\title{
Decision Support Tool for Concurrent Engineering in Space Mission Design
}

\author{
Meenakshi Deshmukh ${ }^{1}$, Volker Schaus ${ }^{1}$, Philipp M. Fischer ${ }^{1}$, Dominik \\ Quantius $^{2}$, Volker Maiwald ${ }^{2}$, Andreas Gerndt ${ }^{1}$
}

\begin{abstract}
The concurrent engineering (CE) approach has been successfully applied to the early design phase of space missions. During CE sessions, a software support is needed to allow multidisciplinary design data exchange. At the moment, a spreadsheet-based solution enhanced with macros is used at the German Aerospace Center (DLR) to create a system model of a space mission during the early design phase. Now there is an increasing demand to take advantage of this system model and provide data analysis features which improve the decision making during CE sessions. Since the current approach is limited for such analysis, DLR has started developing a new tool called Virtual Satellite. It offers extended software support required by the Concurrent Engineering Facility of DLR in Bremen. On top of the previous spreadsheet functionalities, it provides means for online data analysis and system modeling. The results of these data analyses are presented to the discipline experts using different views which help in performing an early design optimization. In this paper, the impact of these views on the decision making during the AEGIS space mission study is presented as a proof of concept.
\end{abstract}

\section{Keywords.}

Concurrent Engineering, Space Missions, Decision Support, Systems Engineering

\section{Introduction}

Over the past ten years, the concurrent engineering (CE) process has been widely used for early space mission design. International and national space agencies as well as industrial organizations have established special CE design centers, such

\footnotetext{
${ }^{1}$ M. Deshmukh ( $\left.₫\right)$,V. Schaus, P. Fischer, A. Gerndt

German Aerospace Center, Simulation and Software Technology, Lilienthalplatz 7, 38108

Braunschweig, Germany

E-Mail: meenakshi.deshmukh@dlr.de

${ }^{2}$ D. Quantius, V. Maiwald

German Aerospace Center, Institute of Space Systems, Robert-Hooke-Str. 7, 28359

Bremen, Germany
} 
as the Concurrent Design Facility of ESA at ESTEC [1] and the Satellite Design Office [2] of EADS Astrium in Friedrichshafen.

The German Aerospace Centre (DLR) has opened its Concurrent Engineering Facility (CEF) at the Institute of Space Systems in Bremen, Germany in 2008 [3]. This facility provides an environment that supports experts from different disciplines to discuss and create a feasible mission design within a couple of weeks. The main goal of carrying out Phase 0/A assessment studies of space missions in a concurrent environment is to enable experts of all involved disciplines to participate in the design process right from the beginning. The scientists start with the system requirements giving a first idea of the investigated system. Iteratively all involved disciplines add information to the design until they find common ground to meet the requirements. The $\mathrm{CE}$ approach has reduced the space mission study duration by a factor of four and study costs by a factor of two. It improves communication between scientists and engineers. It has improved the quality of the results generated in early space mission design phase [1]. In past years a Microsoft Excel ${ }^{\circledR}$ based tool named Integrated Design Model (IDM) has been used in ESA's CDF [4] as well as in DLR's CEF for handling the data that is generated during the study.

IDM does not support distributed work and thus lacks in concurrency. It is difficult to control changes and maintain the model versions with IDM. The IDM does not present the system components structure in a hierarchical manner. Therefore users have to scroll through large data sheets and the comprehensibility is limited. Furthermore the IDM could not support CE sessions of later mission design phases [5].

To overcome IDM limitations, ESA has taken an initiative called Open Concurrent Design Server (OCDS) [6]. OCDS provides standardized data representation and exchange, common design methodologies and clients- server communication via web services. However, it is currently only available within ESA and its partners and not for external projects.

Another tool called MusSat was developed by the Institute of Astronautics at Technical University of Munich to support the model based satellite design process at the Satellite Design Office [7, 2]. It was an academic project and the tool is not commercially available. They have also developed a commercial tool called (v)Sys-ed which is based on an object-oriented principle [8]. It fulfills the basic functionalities required to carry out satellite design phase using CE approach with an addition of data analysis features. It was successfully used in CEF DLR for one study. Because of the licensing issues, the tool was not further used at CEF DLR.

Consequently, DLR decided to develop a new software framework to overcome these known limitations. This framework is called Virtual Satellite which aims to support the space mission design process over the various phases of spacecraft design $[9,10]$. In recent years, the main focus was on the early phases dealing with feasibility studies and preliminary designs, called Phase 0/A. The requirements for the software came mainly from the multi-disciplinary experts who frequently par- 
ticipate in CE activities. Three important aspects for the development were identified during this requirement analysis:

1. To provide same functionality as the existing tool and to overcome its limitations.

2. To incorporate model-based systems engineering, a system data model as well as consistency.

3. To enable a distributed collaborative work environment that allows online and offline work and easy integration of experts being geographically or temporally separated.

The Virtual Satellite was first time used in the CEF for carrying out the telescope space mission AEGIS in December 2011 [11]. During the whole study, the new tool was used for all typical CE tasks like entering relevant system parameters, submitting subsystem designs and receiving input from other disciplines as well as supporting data presentation during moderated sessions and the organization of these. Compared to the previous tools it is evident that additional features for analyzing the design parameters helped to drive the design from session to session.

The paper is organized as follows: Section 2 gives background information about the framework Virtual Satellite and the AEGIS mission. Section 3 presents the purpose and contribution of different data analysis views in the Virtual Satellite and explains their impact on the decision making during the study. The paper ends with the conclusion and insight to the future work in Section 4.

\section{Background}

This section describes the Virtual Satellite and the AEGIS study in detail.

\subsection{Virtual Satellite}

The Virtual Satellite represents a software framework to support the concurrent engineering process for space mission design. It allows all discipline experts attending the CE sessions to enter, store, view and share all design data. Based on Eclipse and Java, it features a modern and extensible framework for CE software. The Eclipse Modeling Framework is used to create the data model contained in the repository [9]. The integration of the Virtual Satellite within the CEF is shown in Figure 1.

Each discipline expert runs an instance of Virtual Satellite on his/her workstation. The software provides them different views containing charts or tables to enter and display the design data. The two main views are the Navigator and the 
Editor. The Navigator view shows a hierarchical tree-like decomposition of the system. Discipline experts can change details by adding, removing or reorganizing the subsystems in the tree. An individual editor can be opened for each subsystem and component. This editor allows attaching parameter information (name, value, unit etc.) to it. Once parameters are defined they can be used in calculations which are also accessible through the editor. On the top system level, an editor allows specifying modes of operation for the whole system. They can be attached individually to each parameter, thus values for each individual mode can be specified [12]. The Virtual Satellite also analyzes design data and presents the results graphically using various views. These views effectively support the design decisions taken during the CE sessions. They are discussed in detail in Section 3.

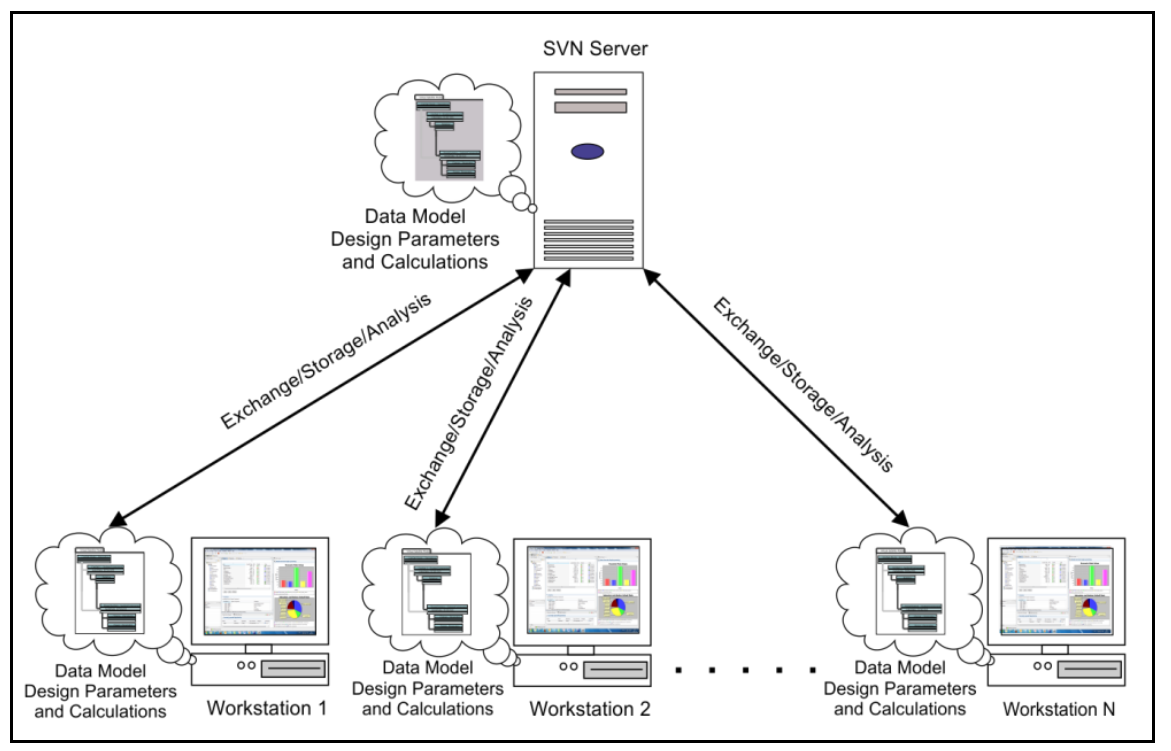

Fig. 1 The general overview of integration of the Virtual Satellite in DLR's CEF in Bremen, Germany

A common repository is created for the persistent and synchronized storage of collected design data using the version control system Subversion (SVN) [13]. A role management feature in the Virtual Satellite organizes access to the individual sub systems of the design. Only the assigned discipline user can alter their design data. This avoids merge conflicts on the SVN backend [9].

To test the software, it has been thoroughly evaluated during its development. Subsequently the Virtual Satellite has been officially applied for the first time in the CEF for carrying out the early design of the astronomy mission AEGIS, thus validating the software in a field test. 


\subsection{AEGIS Study}

The Advanced European Galaxy Imager \& Spectrograph (AEGIS) study is a proposal for a future space mission project which was first presented at the Alpbach Summer School 2011 [11]. The summer school provides a platform for students to practically apply their theoretical knowledge. Based on last year's theme 'Star Formation across the Universe', the AEGIS mission was developed by a group of students supported by several space mission experts including experts from the DLR Institute of Space Systems.

AEGIS is a UV telescope and spectrometer to observe starburst galaxies. To fulfill the scientific goals a big telescope with a diameter of two meters on the main mirror is needed. Furthermore constraints on the attitude and orbit control system had to be obeyed to guarantee pointing stability during the scientific campaign, as well as avoiding direct sun radiation into the telescope. Planning for a lifetime of around 20 to 25 years demands enough propellant for orbit corrections. All together the initial AEGIS design is around $3000 \mathrm{~kg}$ of total mass.

To continue and improve the design of AEGIS, the students from the summer school were invited to the CEF in Bremen, Germany for a one week concurrent engineering study. During this week, they refined various design parameters e.g., dimensions, mass, power, data and temperatures on equipment level and designed the AEGIS satellite in a concurrent environment.

\section{Role of the Virtual Satellite in Decision Support}

As introduced in Subsection 2.1, the design data collected during CE sessions must be interpreted efficiently, in a way that all discipline experts get a thorough understanding of each subsystem and the system as a whole.

Therefore along with data storage and system modeling, the Virtual Satellite also provides means for data analysis and presents the results using different views. All of these provide an improved insight into the overall system and play an important role in making design decisions.

In the following subsections, some of the views of Virtual Satellite are explained in detail with their purpose. The impact of these views in decision making is illustrated with the help of the AEGIS study results. 


\subsection{History View}

The History View is a separate design data analysis tool. It can be configured to display the development of any design parameter over time. In this case, time is represented by revision numbers extracted from the underlying version control system. Figure 2 depicts a history plot as it has been used in the final presentation of the AEGIS study. It shows the development of one important design parameter of the spacecraft, the launch mass. This represents the total mass of the satellite at the time when it is ready to be launched into the orbit. Typically this includes the structural mass and all equipment as well as propellant in tanks and any other additional mass, e.g., adapter to the launcher. The launch mass is plotted over all revisions that have been committed during one week of concurrent engineering sessions. Significant changes in the design can be identified and discussed in the design team if necessary. In this case, the major changes in the design were simply highlighted with handwritten notes. For example, the first drop of the launch mass occurred because the design team found a mistake in the safety margin calculation of the telescope. Such margins are often applied to compensate for uncertainties. The mistake was corrected and the mass was reduced by almost $900 \mathrm{~kg}$. The plot can point out such changes and helps to improve the common understanding amongst the design team.

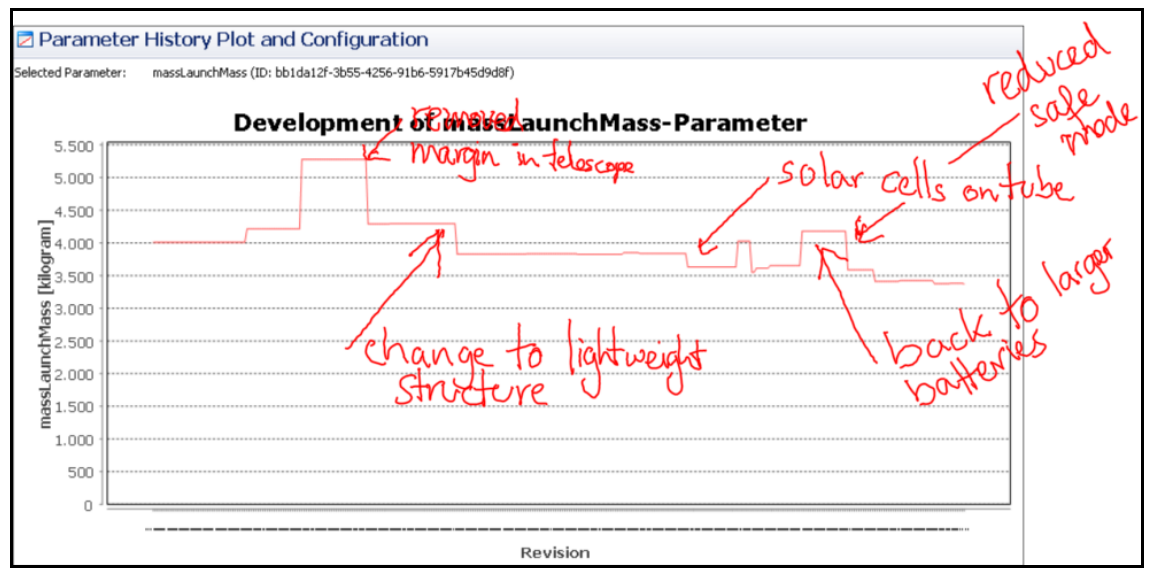

Fig. 2 The history of the launch mass of the AEGIS satellite study. The plot shows major changes of the mass and interpretation (hand-written notes). Usually they indicate important design decisions which were made throughout the week, e.g., the second drop comes from a decision to use a lightweight carbon structure as support for the main mirror of the satellite's telescope

The History View can also be used to document variants of a design. An example of such a case is the second drop of the launch mass which is due to the change of the support structure of the main telescope mirror. At first the support structure was assumed to be a classical aluminum alloy structure. This was then changed to 
a lightweight carbon fiber design to reduce the mass. The lightweight structure is more difficult to build thus makes the satellite more expensive. This is a typical trade-off situation and requires a design decision usually made by the project leader or system engineer. The History View records this decision. Together with the version control, it enables looking at different options of the design starting at a certain revision. The design team can branch at this revision and look into the variant that sticks to the classical aluminum support structure where the baseline of the design continues with the lightweight structure.

Another possibility is that a design flaw is realized and the design team has to go back to a former revision. The History View can help to find the specific revision. Then, the version control is used to roll back the study to the previous date and the team can continue with their design work. The History View is available on all workplaces in the CEF and can be used by any discipline of the design team. The observed parameter can be set to any parameter of the study. Once configured, the history will be created in a separate task running in the background. This means that the user is not blocked and can continue working while the History View is processing in parallel.

\subsection{Summary View}

The Summary View provided by the Virtual Satellite is mainly used by the system engineer and the team leader of a satellite design study. It provides an overview table of the mass of each discipline or subsystem contributing to the total system mass as shown in Figure 3. It calculates and adds margins on different levels and at the very end, gives the total mass of the system at launch and compares it to the maximum capability of the selected launcher of the mission. A pie chart visualizes the mass contribution automatically. This makes it easy for the systems engineer to identify the major contributor, in our case the telescope and all detectors.

Also, these values of the mass budget can be easily compared with the existing satellite missions or heuristics. For example, the core body structure and spacecraft adapter typically account for $10 \%$ - $20 \%$ of the spacecraft's dry mass in case of a small satellite [14]. Looking at the last column in the structure row of the table in Figure 3, the structural contribution to the total dry mass is at $22 \%$ which is slightly higher than the maximum value. The Summary View helps to point out these potential issues. However, it is the responsibility of the team leader to interpret these values and for the team to decide the necessary actions. It might be that the structural contributions for space telescope missions are always a bit higher than on other spacecraft missions. The decision could be to initiate a detailed examination of past telescope missions and assign the task to the structural expert. 


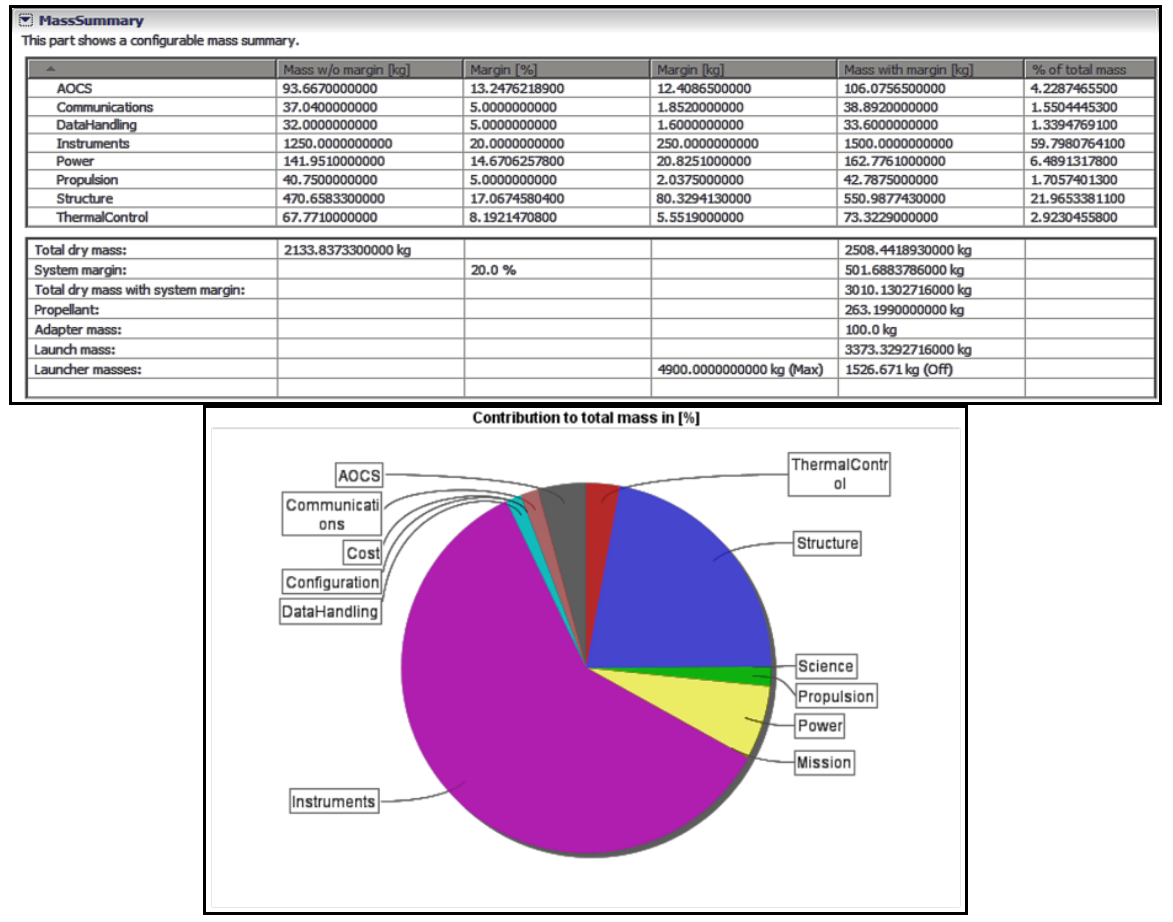

Fig. 3 The Summary View shows the contribution of each discipline to the total mass of the system in a simple table and additionally as pie chart.

\subsection{Mode View}

The Virtual Satellite allows adding modes that describe different operation scenarios during a mission. These modes define a specific state of a system or a subsystem. For example, Safe Mode is a state of a system when only subsystems essential for spacecraft survival are operating. For example, attitude and orbit control subsystem (AOCS) components, communication components etc. Non-essential subsystems are shut down. Science Survey Mode is a time interval when the telescope is carrying out actual measurements. Some design parameters (power, thermal etc.) of subsystems change in each mode. The Virtual Satellite provides the Mode View where the subsystem contribution in different modes is represented using graphs. Such graphical representation provides better insight for systems engineer for analyzing impact of modes on system and subsystem level.

The Mode View in Virtual Satellite contains two types of graphs: bar charts and pie charts. The former is used to display the variation in a parameter value in 
the different modes using a bar chart, the later displays the contribution of each subsystem/discipline in the overall system design in a given mode.

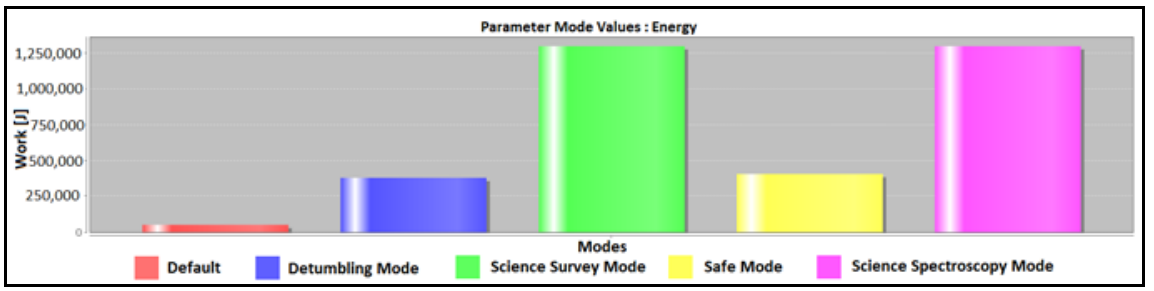

Fig. 4 The Mode View showing overall energy consumption of the telescope for all modes defined in AEGIS mission.

Figure 4 and 5 show examples of the Mode View of Virtual Satellite generated during the AEGIS study. The bar chart in Figure 4 shows the overall energy consumption of the telescope for all modes. As expected, the most demanding situations occur for the two science modes (center and right bar). The pie chart in Figure 5 shows contribution to the average power demand of each discipline in Detumbling Mode and Science Survey Mode respectively. It can be seen that in Detumbling Mode the AOCS has a significant contribution, which is reduced during the Science Survey Mode. During the former, the telescope has no contribution at all, but a significant one in the later mode.

Instead of scrolling through individual discipline data sheets, such graphical representation of data provides easy and efficient insight into mode dependent values, which is especially helpful during early design phases when it is more important to identify design drivers than to generate very precise numerical data. The systems engineer can monitor changes in parameter values in different modes as shown in the bar chart. Both charts together present the mode impact on the individual disciplines as well as on the overall system level. Any changes to the parameter values at discipline level automatically trigger an update of the Mode View.

\subsection{Problem View}

The Virtual Satellite provides the Problem View which gives feedback to the discipline experts about their actions, if error occurs. Whenever they enter data or a calculation, the Virtual Satellite automatically analyzes it in the background against predefined rules. The Problem view displays all the error, warning and information messages generated after data analysis where user attention and actions are required. Figure 6 shows a screenshot of an example of the Problem View taken during the AEGIS study. The user has entered an incorrect calculation which leads to cyclic dependency between an input and output parameter. Similar- 
ly calculation problems like divide by zero, invalid input etc. are immediately detected and the user is informed by displaying corresponding messages in the Problem View. The Problem view also screens warning messages which inform the user about incorrect data. For example, out of bound parameter values. Information messages regarding activities performed by other disciplines are also issued in the Problem View.

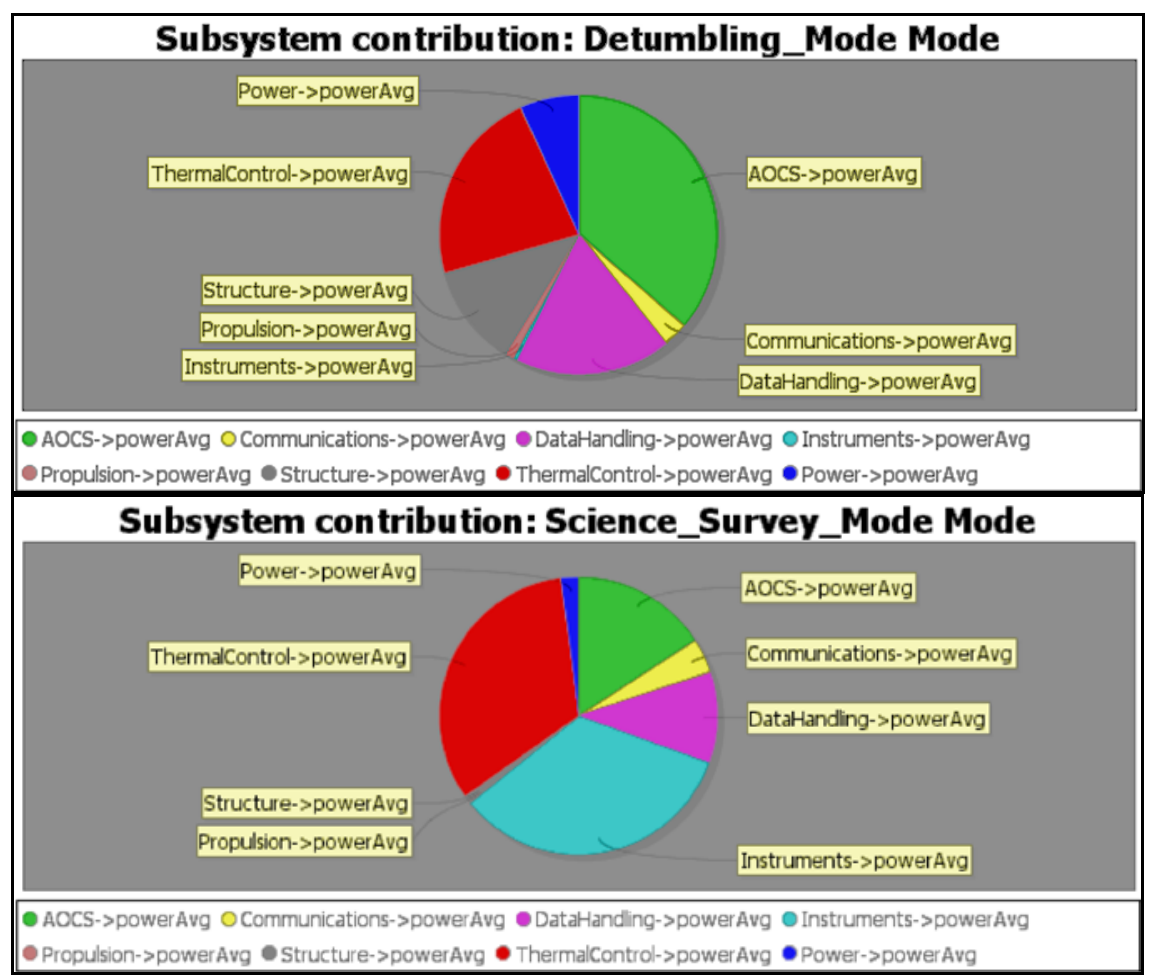

Fig. 5 The Mode View showing average power distribution for each discipline in Detumbling and Science Survey mode in the AEGIS study.

This includes messages stating the addition/deletion of system components or the change in a parameter value. These information messages increase the system awareness of each domain expert. The messages in the Problem View also provide the type and location where user attention is required. Because of these details, a user can easily determine the problem source and what kind of action is needed. 


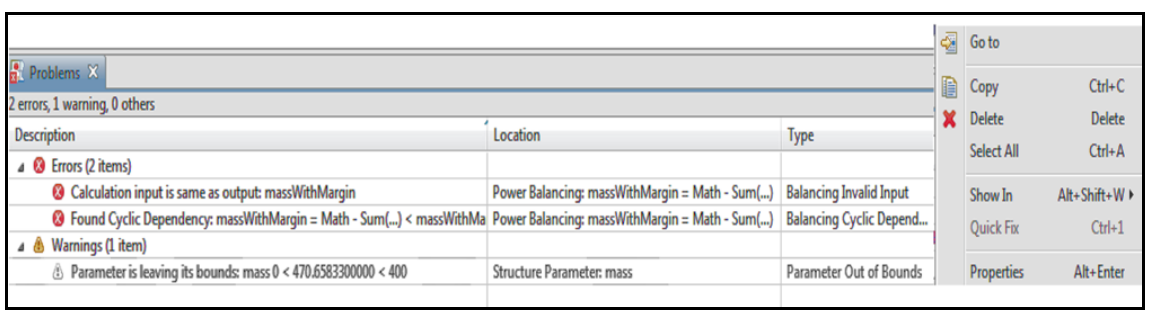

Fig. 6 An example of the Problem View showing error and warning messages during the AEGIS study

Such online feedback assists users in entering data, identify mistakes in early stages and help in preserving data consistency. The other warning and information messages improve user understanding of other subsystems and of system as a whole which results into a good design tradeoff.

\section{Conclusion}

This paper elaborates on the functionalities provided by the software Virtual Satellite in support of the decision making process during early space mission CE design sessions. The Virtual Satellite has successfully provided efficient support during the AEGIS study at the DLR's CEF, by enabling efficient, quick and easy interdisciplinary data exchange, thus creating a spacecraft model containing mass, power and dimension data for each component.

During the study, with the help of the History View the discipline experts were able to track design evolution. Using the Summary View they were able to view the global system display and interdisciplinary structure. The Mode View provided analysis of mode dependent subsystem demands and design parameter variation. Finally the Problem View supplied users with error messages to correct design parameters and calculations. Such immediate availability of the information reduces amount of time necessary to correct the errors and also improves quality of the design.

Overall the Virtual Satellite supplied a manageable structure for the spacecraft design data. Its features provided a valuable visibility to the design data and identification of design problems. This drove the design decisions in a fast and efficient manner. Furthermore the software effectively supports the organization of moderated sessions. Even though the Virtual Satellite was applied in the CEF for the first time, it was reasonably intuitive in use for the users. The Virtual Satellite has proven to be a valuable software support tool for conducting early space mission design phase in CEF.

To further improve the software's support, some more features need to be implemented. This includes addition of sensitivity analysis functions and tools for mission planning. For example, more thorough orbit calculations and visibility to 
discipline dependencies. As the CE approach also has potential to be applied in the later phases (i.e., post-phase-A) of space missions [15], the Virtual Satellite's development will be investigated in this direction as well.

\section{References}

1. The ESA Concurrent Design Facility: Concurrent Engineering applied to space mission assessments. (2011) http://esamultimedia.esa.int/docs/cdf/CDF-INFOPACK-2011.pdf. Accessed 16 January 2012

2. Mager R, Hartmann R (2000) The Satellite Design Office at Astrium - A Success Story of an Industrial Design Center Application. Proceedings of the 2nd Concurrent Engineering Conference (EuSEC), Munich, Germany

3. Romberg O, Braukhane A, Schumann H (2008) Status of the Concurrent Engineering Facility at DLR Bremen. German Aerospace Congress, Dresden, Germany

4. Bandecchi M, Melton B (1999) Concurrent Engineering Applied to Space Mission Assessment and Design. ESA Bulletin

5. Schumann H, Wendel H, Braukhane A, Berres A, Gerndt A, Schreiber A (2010) Concurrent Systems Engineering in Aerospace: From Excel-based to Model Driven Design. 8th Conference on System Engineering Research, Hoboken, NJ, USA

6. Matthyssen A, Bandecchi M (2008) Open Concurrent Design Server Building blocks and Usage, $3^{\text {rd }}$ International Workshop on System \& Concurrent Engineering for Space Applications, SECESA'08, Rome, October 2008

7. Wilke M, Quirmbach M, Igenbergs E (2000) MusSat - A Tool for Satellite Design in Concept Design Centers, Proceedings of the. 2nd European Systems Engineering Conference (EuSEC 2000), Munich, September 2000

8. Schiffner M (2007) A Generalized Systems Architecture Tool for Concurrent Design, ESA $2^{\text {nd }}$ Concurrent Engineering for Space Applications Workshop, Noordwijk, 2007

9. Schaus V, Fischer PM, Luedtke D, Braukhane A, Romberg O, Gerndt A (2010) Concurrent Engineering Software Development at German Aerospace Center -Status and Outlook. 4th International Workshop on System \& Concurrent Engineering for Space Applications.

10. ESA Standard ECSS-M-ST-10C Space project management - Project planning and implementation. (2009)

11. AEGIS: Green Team Final Report (2011) Summer School Alpbach 2011. http://www.summerschoolalpbach.at/docs/2011/presentations/Green_Team_Report.pdf. Accessed 16 January 2012

12. Fischer PM, Schaus V, Gerndt A (2011) Design Model Data Exchange between Concurrent Engineering Facilities by Means of Model Transformation. The 13th NASA-ESA Workshop on Product Data Exchange, Cypress, USA

13. Collins-Sussman B, Fitzpatrick BW, Pilato CM (2011) Version Control with Subversion for Subversion 1.7. http://svnbook.red-bean.com/en/1.7/svn-book.pdf. Accessed 17 January 2012

14. Wertz JR, Larson WJ (1999) Space Mission Analysis and Design. Microcosm Press 401 Coral Circle, E1 Segundo, CA 90245-4622 USA

15. Findlay R, Braukhane A, Schubert D, Pedersen JF, Mueller H, Essmann O (2011) Implementation of concurrent engineering to Phase B space system design. CEAS Space Journal DOI 10.1007/s12567-011-0013-y 